\title{
Adaptación y afrontamiento de personas intervenidas quirúrgica- mente por tumor cerebral: revisión de literatura
}

\author{
Meza-García, Carlos Franciscoi*; Reynaga-Ornelas, Luxana²; Moreno-Pérez, Norma Elvira³
}

\begin{abstract}
RESUMEN
Introducción: El regreso de las personas a la vida diaria después de una cirugía por tumor cerebral impacta en sus dimensiones biológica, social, psicológica, ambiental y espiritual. Objetivo: analizar la evidencia científica actual relacionada al proceso de adaptación y afrontamiento que viven las personas que han sobrevivido a cirugía neurológica debido a una tumoración cerebral. Metodología: Se realizó búsqueda sistemática de la evidencia científica publicada en idioma inglés y español desde el año 2009 al 2017 en bases de datos como PubMed, Scielo y Google Académico, utilizando las palabras clave "adaptación", "afrontamiento", "modelo de Roy", "tumor cerebral" y "cirugía". Los criterios de inclusión fueron de acuerdo a las preguntas planteadas. De un total de 75 artículos, solo 15 reunieron los criterios de inclusión. Resultados: Las revisiones sistemáticas, estudios cuasi-experimentales, cualitativos narrativos y descriptivos, muestran evidencias de deterioro cognitivo, deterioro de la calidad de vida, ansiedad, incertidumbre, limitaciones físicas y estrés, en algunos casos en el largo plazo en pacientes sobrevivientes de tumor cerebral como meningioma y glioma. Un estudio de intervención bajo el modelo de adaptación y afrontamiento se reporta en relación con reportan la calidad de vida. Conclusiones: En la literatura presentan diversas formas de afrontamiento y adaptación a la vida cotidiana posterior a la cirugía, también presentan complicaciones físicas y psicológicas. Se sugiere la realización de estudios que informen las intervenciones de enfermería para brindar un cuidado holístico basado en las vivencias propias de personas que pasan por éstas experiencias.
\end{abstract}

Palabras clave: Neoplasias encefálicas; Adaptación; Afrontamiento (DeCS, BIREME).

¿Universidad de Guanajuato, Campus Celaya-Salvatierra, División de Ciencias de la Salud e Ingenierías, programa del Doctorado en Ciencias de Enfermería. E-mail: cf.meza@ugto.mx

¿2Universidad de Guanajuato, Campus León, División de Ciencias de la Salud, Departamento de Enfermería y Obstetricia Sede León. Guanajuato México.

${ }^{3}$ Universidad de Guanajuato, Campus Celaya-Salvatierra, División de Ciencias de la Salud e Ingenierías, Departamento de Enfermería Clínica. Guanajuato, México.

Recibido: 06/03/2019

Aceptado: 24/06/2019

*Autor para correspondencia

Cómo citar este artículo

Meza-García CF, Reynaga-Ornelas L, Moreno-Pérez, NE. Adaptación y afrontamiento de personas intervenidas quirúrgicamente por tumor cerebral: revisión de literatura. SANUS. 2019;(10): 52-69. [Acceso_____]; Disponible en: mes día año URL 


\section{Adaptação de enfrentamento de pessoas que foram submeti- das à cirurgia de tumor cerebral: revisão da literatura}

ABSTRATO

Introdução: $O$ retorno das pessoas à vida cotidiana após a cirurgia do tumor cerebral afeta sua vida biológica, social, psicológica, ambiental e espiritual. O objetivo deste estudo é avaliar as evidências científicas atuais, relatonadas ao processo de adaptação e enfrentamento vivenciado por pessoas que sobreviverem à cirurgia neurológica por tumor cerebral. Objetivo: Determinar los fatores que desencadeiam violência contra o pessoal de saúde. Metodologia: Uma busca sistemática das evidências científicas publicadas em inglês e espanhol foi realizada de 2009 a 2017 em bases de datos como PubMed, Scielo, e Google Scholar, usando as palavras-chave "adaptação", "lidar", "Modelo de Roy", "tumor cerebral" e "cirurgia". Os critérios de inclusão foram estabelecidos de acordo como as questões posou. Resultados: As revisões sistemáticas, quase-experimentais, narrativas qualitativas e descritivas, apresentam evidências de deterioração cognitiva, deterioração da qualidade de vida, ansiedade, limitações físicas e estresse, em alguns casos a longo prazo em relação aoes sobrevientes de tumor cerebral, como meningiomas e gliomas. Um estudo de intervenção que utilizou o modelo de adaptação e enfrentamento, em relação à melhora da qualidade de vida, é relatado. Conclusões: A literatura mostra diversas forma de adaptação e enfrentamento ao cotidiano após a cirurgia, mostrando tambén complicações físicas e psicológicas. Sugere-se a realização de estudos que informem sobre intervenções de enfermagem necessárias à prestação de cuidados holísticos, como base nas experiências das pessoas que passam por essas experiências.

Palavras chave: Neoplasias cerebrais; adaptação; lidar (DeCS;BIREME)

\section{INTRODUCCIÓN}

Cientos de millones de personas en todo el mundo sufren de trastornos neurológicos, más de 6 millones de personas mueren cada año por accidentes cerebrovasculares, y más del $80 \%$ se producen en países de ingresos bajos o medianos ${ }^{(1)}$. Los tumores cerebrales, representan el $2 \%$, de todas las neoplasias, que incluyen desde lesiones bien diferenciadas y relativamente benignas, como los hemangiomas, hasta lesiones altamente invasivas y diferenciadas como el glioblastoma multiforme ${ }^{(2,3)}$. La tasa de incidencia global de los tumores primarios (por su origen) del sistema nervioso central (SNC), es de 10.82 por cada 100, 000 personas al año(2). En países desarrollados solamente el $14 \%$ de los pacientes diagnosticados con tumores del SNC, tienen una supervivencia de más de 10 años y sólo el 1\% es prevenible (4).

Callista Roy en su modelo describe cuatro modos adaptativos del comportamiento de las personas ante la enfermedad: fisológico, interdependencia, función de rol y autoconcepto. Se enfoca en determinar el nivel de adaptación como integrador, compensador o comprometido. Para ella la adaptación es un proceso por el que los pensamientos y los sentimientos eligen la conciencia consciente para crear una integridad humana individua|(5,6). Por lo tanto, es necesario conocer el proceso complejo de adaptación que incluye la integración fisiológica, psicológica y social de las personas.
El objetivo de esta revisión fue conocer cómo la persona sometida a una cirugía neurológica se adapta y afronta a su vida cotidiana.

\section{METODOLOGÍA}

Se desarrollaron algunas preguntas antes de realizar la búsqueda de artículos, ¿Cómo es la adaptación y afrontamiento del paciente sometido a cirugía de tumor cerebral? ¿cuál es el modo adaptativo del paciente con tumor cerebral sometido a cirugía cerebral? ¿Cuál es el proceso de adaptación según el modelo de Roy en los pacientes sometidos a cirugía de tumor cerebral? ¿cómo se da el proceso de adaptación, modo, autoconcepto, en personas con tumor cerebral que sobreviven a la cirugía?

\section{Estrategias de búsqueda}

Se realizó una búsqueda en bases de datos como PubMed, Scielo, Google Académico.

Primera búsqueda con operadores boleanos y palabras claves: Roy Model and adaptation and brain, notchildren, notpediatric, not digital. En el año: 2009-2017. Con un total de 20 artículos seleccionados, de los cuales 8 que incluyen los criterios de inclusión, sólo 6 de lectura y que aportan para la revisión de literatura. 
En la segunda búsqueda las palabras claves con los operadores boleanos fueron coping and adaptation, brain tumor, en el año 2014-2017, con total de 49 artículos. Al realizar el filtro con notpediatric, notcaregiver and notdigital, quedando 7 artículos para la revisión.

En la tercera búsqueda, las palabras claves y operadores boleanos fueron: coping, adaptation, brain tumor, notpediatric, notcaregiver, notdigital, notaucustic, año 2017, con un total de 6 artículos incluidos de los cuales solo dos elegidos para la revisión de literatura. Con un total de 15 artículos.

\section{Descripción de los artículos}

Del total de 75 artículos encontrados sólo 15 fueron incluidos acuerdo a los criterios de inclusión, pregunta de inicio, bases de datos, año y palabras claves. El tipo de estudio de éstos artículos fue: descriptivos/observacional analítico $(n=6)$, revisión sistemática $(n=3)$, cuasi-experimental $(n=$ 1), cualitativo $(n=3)$, experimental $(n=1)$. El idioma en 14 artículos fue el inglés, sólo uno del idioma turco. El origen del artículo fue en Países Bajos ( $n=4)$, Turquía $(n=4)$, EUA $(n=3)$, Australia $(n=1)$, Inglaterra $(n=1)$, Alemania $(n=$ 1) y China $(n=1)$. El año del artículo varía de acuerdo a la búsqueda, del 2014 al 2017 con 11 artículos, 2009 al 2013 con 4 artículos.

\section{RESULTADOS}

Según el estudio cuasi-experimental revisado, Baksi y Dicle ${ }^{(7)}$ en un estudio comparativo de 95 pacientes (control $n=50$; experimental $n=45$ ) se comprobaron el impacto de una intervención educativa basada en el modelo de Roy con un mejor afrontamiento al estrés en los pacientes con tumor cerebral del grupo experimental $(p=0.05)$.

El estudio experimental fue incluido principalmente porque Aramesh y colaboradores ${ }^{(8)}$, reportan que el Modelo de Adaptación de Roy utilizado para evaluar las consecuencias de los pacientes con trauma neurológico es mejor que el índice ESI.

Sobre las revisiones sistemáticas se menciona la de Ownswwort y cols. ${ }^{(9)}$, quienes reportan que en solo 10 de 17 estudios en total demuestran el impacto de la psicoterapia, el apoyo basado en la familia, la rehabilitación cognitiva y las intervenciones basadas en actividades sobre el autoconcepto de personas con lesiones cerebrales traumáticas; concluyen que se reporta pérdida de independencia, pérdida de uno mismo y pérdida de relaciones. Según los sujetos, la información fue útil para el reconocimiento de su experiencia y para la identificación de la necesidad de mayores servicios psicológicos para las personas que presentan tumor cerebral. Así mismo, encontraron que hay mejoría en el autoconcepto con los grupos pre y post al realizarles una intervención de rehabilitación en pacientes con lesión cerebral.

En otra revisión sistemática sobre funcionamiento cognitivo en pacientes con meningioma, Meskal y cols. ${ }^{(10)}$ incluyeron 11 artículos concluyendo que las personas con meningiomas ya presentan deterioro cognitivo anterior a la cirugía (memoria, atención y funciones ejecutivas); la función cognitiva mejora levemente después de la cirugía. Los resultados generales son inconsistentes sobre el funcionamiento cognitivo antes de la cirugía y el mejoramiento posterior.

Zmanipoor Najafbadi (11) en una revisión sistemática reporta 19 artículos y concluye que la calidad de vida en personas con meningioma es menor a los controles sanos. Aunque los pacientes con meningioma tuvieron una mejor calidad de vida que los de glioma, esta diferencia no fue significativa clínicamente. Aunque la resección del tumor mejora la calidad de vida, ésta se muestra persistentemente baja a largo plazo comparada con los controles sanos.

En los estudios cualitativos, Simsek y Dicle (12) examinaron los modos adaptativos de los pacientes con tumor cerebral primario según el Modelo de Adaptación de Roy, realizado a 17 personas una entrevista semiestructurada. Las categorías que se encontraron en el modo fisiológico fueron: efectos secundarios de los fármacos, exposición a las actividades de la vida diaria, fatiga, dolor de cabeza, trastornos visuales y problemas neurológicos; en el modo de autoconcepto describieron estrés causado un sentimiento de malestar por un futuro incierto; en el modo de función del rol, los problemas están relacionados por no poder cumplir con su rol de género y la exposición a tener otras relaciones. Por su parte, Wenström y colaboradores ${ }^{(13)}$, en un estudio cualitativo narrativo describen las experiencias de mujeres después la cirugía de meningioma y su significado; las categorías que se encontraron fueron amenaza doble: tumor y operación, el tumor benigno como amenazante, la cirugía como curativa y arriesgada, la herida como una puerta abierta, el cuerpo sufriente que desborda la vida, mujeres que no pudieron recuperar su cuerpo, sensación de fragilidad y la hipersensibilidad a la impresión. Lucas (14) por su parte, publicó en el 2010 el análisis cualitativo de las entrevistas de cientos de pacientes que atendieron a su clínica de onco-neurología resultando tres principales temas: pérdida de la independencia (física y financiera), pérdida de sí mismo (sentido del humor, personalidad) y pérdida de las relaciones (amigos, trabajo y familia).

Dentro de los estudios observacionales analíticos, Waagemans y cols. ${ }^{(15)}$ incluyeron a 89 pacientes con meningioma midiendo el impacto a largo plazo del déficit cognitivo y la epilepsia en la calidad de vida. No se reportan diferencias con los controles sanos en 7 de 8 escalas del instrumento de Calidad de Vida, el SF36; la única diferencia fue que los pacientes con meningioma informaron más limitaciones por problemas físicos $(p<0,5)$. Además Habets y cols. (16), también midieron el impacto del daño cognitivo en 62 pacientes con glioma de alto grado en el funcionamiento de la vida diaria obteniendo que el $79 \%$ de ellos ya tienen un daño cognitivo antes de la cirugía cuando menos en un dominio. Posterior a la cirugía estudiaron a 39 pacientes encontrando que el 59\% tenían daño cognitivo. En el 
seguimiento el 49\% mostró mejoría mientras que el $23 \%$ empeoró.

Wolters Gregorio(17) por su parte, examinó la relación entre funcionamiento ejecutivo, afrontamiento, síntomas depresivos y calidad de vida en personas con síntomas neuropsiquiátricos con daño cerebral adquirido. Reporta que las personas con disfunción ejecutiva eran aquéllas con los estilos de afrontamiento pasivo $(\beta=.37, p<.01)$, y éste a su vez relacionado con menor calidad de vida ( $\beta$ $=-.57, p<.001)$ y mayor número de síntomas depresivos $(\beta=.65, p<.001)$. Por otra parte, aquéllos pacientes con un estilo de afrontamiento enfocado al problema, tuvieron un mejor funcionamiento ejecutivo $(\beta=.94, p<.05)$. Relacionado con ello, es el estudio realizado en China por Bao y colaboradores ${ }^{(18)}$ donde reportan una prevalencia de 42.8\% de ansiedad y 32.4\% de depresión en 222 pacientes con tumores del sistema nervioso central encuestados y proponen el desarrollo de intervenciones para promover la esperanza y el optimismo basadas en las necesidades específicas de los pacientes. En este sentido, Holmes y colaboradores $^{(19)}$ han demostrado que los desafíos psicológicos de los pacientes con tumores del sistema nervioso central (adaptación, problemas mentales, duelo, incertidumbre, entre otros) requieren de un psiquiatra de contacto para mejorar el manejo de los pacientes y llevar una mejor comunicación con el equipo de salud.

Lin $\operatorname{Lin}^{(20)}$ en un estudio reportado en 2014 con 186 pacientes con tumor cerebral primario en diferentes etapas enfermedad, menciona que el nivel de incertidumbre de los pacientes con tratamiento activo fue tan alto como el de aquellos que son recientemente diagnosticados. El predictor más alto de incertidumbre fue el cambio en el estatus de empleo relacionado con la enfermedad. Esto habla de la necesidad de que los pacientes adquieran habilidades de afrontamiento para manejar la incertidumbre.

Por otro lado Krupp y cols. (21) en un estudio retrospectivo en pacientes posterior a 15 meses ( \pm 3.6) de una cirugía de meningioma supratentorial, encontraron una correlación negativa entre edad y desempeño cognitivo $(p<$ 0.001). El 73\% de los pacientes más jóvenes refirieron no estar satisfechos con la vida, el 68\% reportaron su inhabilidad para aceptar la enfermedad. Además, los pacientes solteros tuvieron una mayor frecuencia de afrontamiento depresivo $(p<0.05)$ y menor satisfacción con la vida $(p<0.05)$.

Los artículos mencionados se encuentran en la tabla 1 se presentan por orden de búsqueda.

\section{DISCUSIÓN}

En base a la pregunta realizada para la búsqueda: ¿Cómo es la adaptación y afrontamiento del paciente sometido a cirugía de tumor cerebral? y ¿cuál es el modo adaptativo del paciente con tumor cerebral sometido a cirugía cerebral? Se encontraron diferentes categorías relacionadas a las respuestas de afrontamiento y adaptación de personas intervenidas de un tumor cerebral y cómo son evaluadas por los autores.
La primera fue calidad de vida. En esta revisión de literatura se encontró que la calidad de vida, se reporta menor en las personas con meningioma que los controles sanos incluso posterior a la resección; además aunque los pacientes con meningioma tuvieron una mejor calidad de vida que los de glioma, esta diferencia no fue significativa clínicamente ${ }^{(11)}$. Se reporta baja calidad de vida en diferentes funcionamientos y asociación entre funcionamiento ejecutivo, psicosocial y afrontamiento después de adquirir daño cerebral. El afrontamiento pasivo está relacionado con una menor calidad de vida ${ }^{(17)}$. La limitación física se encuentra implicada en pacientes con tumores cerebrales, así como la epilepsia a largo plazo, por lo cual se ve afectada la calidad de vida a largo plazo(15).

Ansiedad y la depresión. Un estudio reporta una prevalencia de $42.8 \%$ de ansiedad y $32.4 \%$ de depresión en 222 pacientes con tumores del sistema nervioso central encuestados. La correlación de Pearson, mostró que la ansiedad estaba relacionada con la esperanza $(p<0.01)$, el optimismo $(p<0.01)$ y la autoeficacia general $(p<0.01){ }^{\text {(18) }}$. Posterior a la cirugía, los pacientes solteros tuvieron una mayor frecuencia de afrontamiento depresivo $(p<0.05)$ y menor satisfacción con la vida $(p<0.05)^{(21)}$.

Deterioro cognitivo. Existe deterioro cognitivo en diferentes tumores cerebrales, y desde antes de la cirugía, sin embargo resultados generales son inconsistentes sobre el funcionamiento cognitivo antes de la cirugía y el mejoramiento posterior ${ }^{(10)}$. El nivel cognitivo en personas con tumores cerebrales en este caso gliomas, también la epilepsia implica deterioro cognitivo. Lin en su intervención reporta incertidumbre en pacientes con tumores cerebrales como ambigüedad, incoherencia, imprevisibilidad, y otros factores desencadenantes fueron diferentes entre los grupos de tratamiento $(p=0.01)^{(20)}$. Otros mencionan el $79 \%$ de las personas con glioma ya tienen un daño cognitivo antes de la cirugía cuando menos en un dominio. Posterior a la cirugía estudiaron a 39 pacientes encontrando que el 59\% tenían daño cognitivo. En el seguimiento el 49\% mostró mejoría mientras que el $23 \%$ empeoró(16).

Problemas físicos. En un estudio de personas con meningiomas en Países Bajos en el 2011, no difieren de los controles sanos en 7 de 8 escalas de SF36, la única diferencia fue que los pacientes informaron más limitaciones por problemas físicos $(p<0.5)^{(15)}$.

Secuelas neurológicas varias. Las categorías en estudios cualitativos fueron de efectos secundarios de los fármacos, influencias en las actividades de la vida diaria, fatiga, dolor de cabeza, trastornos visuales y neurológicos ${ }^{(12)}$. La adaptación, los problemas mentales, el duelo, y la incertidumbre, entre otros se presentan en los pacientes con tumores en el sistema nervioso central requiriendo de un psiquiatra de contacto para mejorar el manejo de los pacientes ${ }^{(19)}$.

Percepciones y significados. En las narrativas los pacientes han descrito el tumor benigno como amenazante, la cirugía como curativa y arriesgada. La herida como 
Tabla 1. Características de los artículos seleccionados en esta revisión de literatura

\begin{tabular}{|c|c|c|c|c|c|}
\hline Título, autores y país & Revista y Año & $\begin{array}{l}\text { Tipo de es- } \\
\text { tudio }\end{array}$ & Muestra & Instrumento & Resultados \\
\hline $\begin{array}{l}\text { Deterioro de la calidad } \\
\text { de vida relacionada a la } \\
\text { salud en el meningioma: } \\
\text { revisión sistemática. } \\
\text { Zmanipoor, A., cols. } \\
\text { Países Bajos. }\end{array}$ & Neuro-oncology 2016. & $\begin{array}{l}\text { Revisión siste- } \\
\text { mática. }\end{array}$ & $\begin{array}{l}19 \text { artículos de } \\
\text { acuerdo a los crite- } \\
\text { rios de inclusión. }\end{array}$ & $\begin{array}{l}\text { Búsqueda sistemática: } \\
\text { Palabras clave: Menin- } \\
\text { gioma, Calidad de vida. }\end{array}$ & $\begin{array}{l}\text { Pacientes con meningioma } \\
\text { tienen mejor calidad de } \\
\text { vida que los de glioma, } \\
\text { aunque esta diferencia no } \\
\text { fue clínicamente significa- } \\
\text { tiva }{ }^{(11)} \text {. }\end{array}$ \\
\hline $\begin{array}{l}\text { Funcionamiento cog- } \\
\text { nitivo en pacientes con } \\
\text { meningioma: revisión } \\
\text { sistemática. } \\
\text { Meskal, I., Gehring, K., } \\
\text { Rutten, G., SitsKoorn M. } \\
\text { Países Bajos. }\end{array}$ & $\begin{array}{l}\text { J Neuro-Oncology } \\
2016 .\end{array}$ & $\begin{array}{l}\text { Revisión siste- } \\
\text { mática. }\end{array}$ & $\begin{array}{l}11 \text { artículos } \\
\text { incluyeron dentro } \\
\text { de los criterios de } \\
\text { inclusión. }\end{array}$ & $\begin{array}{l}\text { Base de datos electró- } \\
\text { nica: Pubmed. }\end{array}$ & $\begin{array}{l}\text { Las personas con me- } \\
\text { ningiomas, anterior a } \\
\text { la cirugía ya presentan } \\
\text { deterioro cognitivo (me- } \\
\text { moria, atención, funciones } \\
\text { ejecutivas), el cual mejora } \\
\text { levemente desupés de la } \\
\text { cirugía. No hay consisten- } \\
\text { cia ente los resultados de } \\
\text { los estudios(10) }\end{array}$ \\
\hline $\begin{array}{l}\text { Impacto a largo plazo } \\
\text { del déficit cognitivo y la } \\
\text { epilepsia en la calidad } \\
\text { de vida en paciente con } \\
\text { meningioma. } \\
\text { Waagemans, M. y cols. } \\
\text { Países Bajos. }\end{array}$ & $\begin{array}{l}\text { Neuro-surgery-online. } \\
2011\end{array}$ & $\begin{array}{l}\text { Observacional } \\
\text { analítico. }\end{array}$ & 89 pacientes. & $\begin{array}{l}\text { Neuropsychological } \\
\text { test battery }\end{array}$ & $\begin{array}{l}\text { Los pacientes con menin- } \\
\text { giomas no difieren de los } \\
\text { controles sanos en } 7 \text { de } 8 \\
\text { escalas de SF36, la única } \\
\text { diferencia fue que los } \\
\text { pacientes informaron más } \\
\text { limitaciones por problemas } \\
\text { físicos }(p<0.5)^{(15)} \text {. }\end{array}$ \\
\hline $\begin{array}{l}\text { Revisión de los estados } \\
\text { de adaptación de los } \\
\text { pacientes con tumor } \\
\text { cerebral primario según } \\
\text { el modelo de adapta- } \\
\text { ción de Roy: un análisis } \\
\text { cualitativo. } \\
\text { Simsek, A., Dicle, A. } \\
\text { Turquía. }\end{array}$ & $\begin{array}{l}\text { Journal of Neurological } \\
\text { Sciences. }\end{array}$ & Cualitativa. & 17 pacientes. & $\begin{array}{l}\text { Entrevista a profundi- } \\
\text { dad. } \\
\text { Entrevista semi-estruc- } \\
\text { turada. }\end{array}$ & $\begin{array}{l}\text { Efectos secundarios de los } \\
\text { fármacos. Influencias en } \\
\text { las actividades de la vida } \\
\text { diaria. Fatiga, dolor de } \\
\text { cabeza, trastornos visuales, } \\
\text { neurológicos. Función del } \\
\text { rol: rol de género. Función } \\
\text { de autoconcepto: sentirse } \\
\text { mal y sobre el futuro (12). }\end{array}$ \\
\hline $\begin{array}{l}\text { Vivir en una paradoja, } \\
\text { experiencias de mujeres } \\
\text { en el mundo después la } \\
\text { cirugía de meningioma. } \\
\text { Wenström, I, Eriksson, } \\
\text { LE. Ebbeskog, B. Ingla- } \\
\text { terra. }\end{array}$ & $\begin{array}{l}\text { Journal of advanced } \\
\text { nursing, } 2012 \text {. }\end{array}$ & $\begin{array}{l}\text { Cualitativo, } \\
\text { Narrativo. }\end{array}$ & 6 personas & $\begin{array}{l}\text { Entrevista. } \\
\text { Observacional. }\end{array}$ & $\begin{array}{l}\text { Amenaza doble: tumor } \\
\text { y operación, el tumor } \\
\text { benigno como amenazan- } \\
\text { te, la cirugía como curativa } \\
\text { y arriesgada, la herida } \\
\text { como una puerta abierta, } \\
\text { el cuerpo sufriente que } \\
\text { desborda la vida, las muje- } \\
\text { res no pudieron recuperar } \\
\text { su cuerpo, sensación de } \\
\text { fragilidad y la hipersensibi- } \\
\text { lidad a la impresión }{ }^{(13)} \text {. }\end{array}$ \\
\hline
\end{tabular}




\begin{tabular}{|c|c|c|c|c|c|}
\hline $\begin{array}{l}\text { Tumor y efectos de la } \\
\text { de la cirugía en función } \\
\text { cognitiva en pacientes } \\
\text { con gliomas de alto } \\
\text { grado. } \\
\text { Habets, E., Kloet, A., } \\
\text { Walchemback, R., Vech, } \\
\text { C., Klein, M. and Ta- } \\
\text { phoorn. Países Bajos. }\end{array}$ & Acta Neurochir, 2014. & $\begin{array}{l}\text { Observacional } \\
\text { analítico } \\
\text { Cohorte. }\end{array}$ & $\begin{array}{l}\text { Control: } 31 . \\
\text { Seguimiento: } 62 . \\
\text { Total: } 93 .\end{array}$ & $\begin{array}{l}\text { Test de dominos cog- } \\
\text { nitivos. }\end{array}$ & $\begin{array}{l}\text { Comparado con los } \\
\text { controles sanos, la función } \\
\text { cognitiva de los pacientes } \\
\text { está disminuida signfi- } \\
\text { cativamente en todos los } \\
\text { dominios. Después de un } \\
\text { seguimiento de } 5 \text { semanas } \\
\text { después de la cirugía, } \\
\text { el } 59 \% \text { de los pacientes } \\
\text { tenían una disfunción } \\
\text { cognitiva al menos en un } \\
\text { dominio. En el seguimien- } \\
\text { to el } 49 \% \text { mostró mejoría } \\
\text { y el } 23 \% \text { empeoró(16). }\end{array}$ \\
\hline $\begin{array}{l}\text { Enlace Psiquiátrico del } \\
\text { servicio de tumores del } \\
\text { sistema nervioso central. } \\
\text { Holmes, A., Adams, } \\
\text { S., Hall, S., Roshental, } \\
\text { M. and Drummond, K. } \\
\text { Australia. }\end{array}$ & Neuro-Oncology, 2015. & $\begin{array}{l}\text { Descriptivo, } \\
\text { retrospectivo. }\end{array}$ & $\begin{array}{l}\text { Análisis de } 5 \text { años } \\
\text { de problemas } \\
\text { experimentados } \\
\text { por los pacientes } \\
\text { y su manejo por el } \\
\text { psiquiatra. }\end{array}$ & $\begin{array}{l}\text { Variables socio-demo- } \\
\text { gráficas. } \\
\text { Tipos de tumores, } \\
\text { Desafío psicológico, } \\
\text { Diagnósticos psiquiá- } \\
\text { tricos. }\end{array}$ & $\begin{array}{l}\text { Frecuencias y porcentajes } \\
\text { para todas las variables }{ }^{(19)} \text {. }\end{array}$ \\
\hline $\begin{array}{l}\text { Asociación entre funcio- } \\
\text { namiento ejecutivo, psi- } \\
\text { cosocial y afrontamiento } \\
\text { después de adquirir } \\
\text { daño cerebral. } \\
\text { Wolters Gregorio, y } \\
\text { cols. } \\
\text { Países Bajos. }\end{array}$ & $\begin{array}{l}\text { British Journal and Cli- } \\
\text { nic Psychology Society } \\
2015 .\end{array}$ & $\begin{array}{l}\text { Observacional } \\
\text { correlacional. } \\
\text { Longitu }\end{array}$ & $\begin{array}{l}\text { Primera muestra: } \\
93 \\
\text { Segunda muestra: } \\
58\end{array}$ & $\begin{array}{l}\text { 1.- Stroop color word } \\
\text { test. 2.- Frontal systems } \\
\text { behavioral scale. 3.- } \\
\text { Ultrecht Coping List. } \\
\text { 4.- Cuestionario de } \\
\text { salud del paciente. } \\
\text { 5.- Cuestionario de } \\
\text { satisfacción de la vida. }\end{array}$ & $\begin{array}{l}\text { Disfunción ejecutiva se } \\
\text { asocia con mayor uso de } \\
\text { estilos de afrontamiento } \\
(p=0,01) \text {. El afrontamiento } \\
\text { pasivo se asocia con baja } \\
\text { calidad de vida }(p=0,001) \\
\text { y síntomas depresivos } \\
(p=0,001)(17) \text {. }\end{array}$ \\
\hline $\begin{array}{l}\text { Prevalencia y variables } \\
\text { psicológicas positivas } \\
\text { asociadas con ansiedad } \\
\text { y depresión entre } \\
\text { pacientes con tumor del } \\
\text { sistema nervioso central } \\
\text { en China } \\
\text { Bao, Y., y cols. } \\
\text { China. }\end{array}$ & $\begin{array}{l}\text { Psycho-Oncology } \\
2017\end{array}$ & $\begin{array}{l}\text { Observacional } \\
\text { analítico. }\end{array}$ & $\mathrm{N}=222$ personas. & $\begin{array}{l}\text { 1.- Escala de Ansiedad } \\
\text { y Depresión. } \\
\text { 2.- Indice de Herth y } \\
\text { Hope. } \\
\text { 3.- Escala de Orienta- } \\
\text { ción, revisada. } \\
\text { 4.- Escala de autoefica- } \\
\text { cia general. } \\
\text { 5.- Demografía y } \\
\text { síntomas clínicos con } \\
\text { tumores del SNC. }\end{array}$ & $\begin{array}{l}\text { La educación tuvo un } \\
\text { efecto significativo en la } \\
\text { puntuación de ansiedad } \\
(p=0,005) \text { y depresión } \\
(p=0,001) \text {. } \\
\text { correlación de Pearson, } \\
\text { mostró que la ansiedad } \\
\text { estaba negativamente } \\
\text { relacionada con la espe- } \\
\text { ranza }(r=0,46, p<0,01) \text {, op- } \\
\text { timismo }(r=0,39, p<0,01) \\
\text { y autoeficacioa general } \\
(r=0,21, p<0,01) \text { (sis). }\end{array}$ \\
\hline $\begin{array}{l}\text { Predictores significativos } \\
\text { de incertidumbre en } \\
\text { paciente con tumores } \\
\text { cerebrales. } \\
\text { Lin, L., Chien, L., Acqua- } \\
\text { ye, A., Vera, E., Gilbert, } \\
\text { M., Amstrong, T. EUA }\end{array}$ & J Neuroncolgy. 2015 & $\begin{array}{l}\text { Descriptivo, } \\
\text { observacional }\end{array}$ & $\mathrm{N}=186$ pacientes & $\begin{array}{l}\text { 1.- Mishel Uncertainty } \\
\text { in Illness Scale. } \\
\text { 2.- Demographic } \\
\text { Information Sheet. } \\
\text { 3.- Clinical Assessment } \\
\text { Tool. }\end{array}$ & $\begin{array}{l}\text { Los predictores en total } \\
\text { tres sub escalas (ambi- } \\
\text { güedad, incoherencia, } \\
\text { imprevisibilidad, y otros } \\
\text { factores desencadenantes) } \\
\text { fueron diferentes entre } \\
\text { los grupos de tratamiento } \\
(p=0,01)^{(20)} \text {. }\end{array}$ \\
\hline
\end{tabular}




\begin{tabular}{|c|c|c|c|c|c|}
\hline $\begin{array}{l}\text { Efecto de una inter- } \\
\text { vención utilizando el } \\
\text { modelo de Roy en } \\
\text { pacientes con tumores } \\
\text { primarios en síntomas y } \\
\text { adaptacíón al estrés. } \\
\text { Baksi A., Dicle, A. } \\
\text { Turquía. }\end{array}$ & $\begin{array}{l}\text { International Journal of } \\
\text { Caring Science, } 2017 .\end{array}$ & $\begin{array}{l}\text { Intervención: } \\
\text { Cuasi-experi- } \\
\text { mental }\end{array}$ & $\begin{array}{l}95 \text { pacientes } \\
\text { Control } \\
\text { Experimental }\end{array}$ & $\begin{array}{l}\text { 1.- Karnofsky perfor- } \\
\text { mance status. } \\
\text { 2.- MD Anderson } \\
\text { Symptom inven- } \\
\text { tory-brain tumor } \\
\text { Turkish. } \\
\text { 3.- Ways of Coping with } \\
\text { Stress Scale. }\end{array}$ & $\begin{array}{l}\text { Hay diferencia significativa } \\
\text { entre el grupo control y } \\
\text { experimental tomando el } \\
\text { valor estadístico }(p=0,05)^{(7)} \text {. }\end{array}$ \\
\hline $\begin{array}{l}\text { Implicaciones psicoso- } \\
\text { ciales con el paciente } \\
\text { con glioma de alto } \\
\text { grado. } \\
\text { Michel R. Lucas. EUA. }\end{array}$ & $\begin{array}{l}\text { Journal of Neurocience } \\
\text { Nursing, } 2010 .\end{array}$ & $\begin{array}{l}\text { Cualitativo, } \\
\text { experiencias. }\end{array}$ & $\begin{array}{l}\text { Periodo de } 7 \text { años } \\
\text { de 2001-2008. }\end{array}$ & $\begin{array}{l}\text { Entrevista semiestruc- } \\
\text { turada. }\end{array}$ & $\begin{array}{l}\text { Tres categorías: pérdida de } \\
\text { independencia, pérdida de } \\
\text { uno mismo y pérdida de } \\
\text { relaciones }{ }^{(14)} \text {. }\end{array}$ \\
\hline $\begin{array}{l}\text { Impacto de la rehabi- } \\
\text { litación en el autocon- } \\
\text { cepto, lesión cerebral } \\
\text { traumática: una revisión } \\
\text { sistemática exploratoria } \\
\text { de la de la metodología, } \\
\text { intervención y eficacia. } \\
\text { Ownsworth, T. y Haslam, } \\
\text { C. Australia. }\end{array}$ & $\begin{array}{l}\text { Neuropsychological } \\
\text { Reabilitation. } \\
2014 .\end{array}$ & $\begin{array}{l}\text { Revisión siste- } \\
\text { mática. }\end{array}$ & $\begin{array}{l}17 \text { estudios en base } \\
\text { de datos. }\end{array}$ & Palabras clave. & $\begin{array}{l}\text { Solo } 10 \text { artículos se } \\
\text { encontraron mejoría en } \\
\text { el auto-concepto con los } \\
\text { grupos pre y post }{ }^{(9)} \text {. }\end{array}$ \\
\hline $\begin{array}{l}\text { Comparando la eficacia } \\
\text { de la atención de } \\
\text { enfermería basada en } \\
\text { ESI, Triage y modelo de } \\
\text { adaptación de Roy para } \\
\text { predecir la necesidad } \\
\text { de una UCI o sala de } \\
\text { admisión entre los pa- } \\
\text { cientes con traumatismo } \\
\text { craneal. } \\
\text { Aramesh Z., Mazloum } \\
\text { R., Aghebati N. y Mass- } \\
\text { hadiNejad, H. Turquia. }\end{array}$ & $\begin{array}{l}\text { Journal of Manzan- } \\
\text { daran University of } \\
\text { Medical Science. } \\
\text { 2017. }\end{array}$ & Experimental & $\begin{array}{l}400 \text { personas, } \\
\text { doscientos para } \\
\text { cada grupo. }\end{array}$ & $\begin{array}{l}\text { ESI (Emergency severity } \\
\text { index) Triage. } \\
\text { Modelo de Roy }\end{array}$ & $\begin{array}{l}\text { Comparado con el índice } \\
\text { ESI, el Modelo de Adapta- } \\
\text { ción de Roy podría resultar } \\
\text { en menos errores para } \\
\text { predecir las consecuen- } \\
\text { cias de los pacientes con } \\
\text { trauma neurológico(8) }\end{array}$ \\
\hline $\begin{array}{l}\text { Evaluación de los pará- } \\
\text { metros neuropsicológi- } \\
\text { cos calidad de vida para } \\
\text { evaluar los resultados } \\
\text { con pacientes con } \\
\text { meningioma supraten- } \\
\text { torial, tratado quirúrgi- } \\
\text { camente. } \\
\text { Krupp W. y cols. Ale- } \\
\text { mania. }\end{array}$ & Neurosurgery. 2009. & $\begin{array}{l}\text { Observacional } \\
\text { analítico }\end{array}$ & 91 Pacientes & $\begin{array}{l}\text { 1.- Test of Attention. } \\
\text { 2.- LPS Intelligence Test. } \\
\text { 3.- Freiburg Question- } \\
\text { naire of Coping with } \\
\text { Illness. }\end{array}$ & $\begin{array}{l}\text { Hay significancia negativa } \\
\text { de correlación entre la } \\
\text { edad del paciente y el } \\
\text { rendimiento cognitivo } \\
(p=0,001) \text {. Los pacientes } \\
\text { que viven solteros tienen } \\
\text { una mayor frecuencia de } \\
\text { depresión }(p=0,05)^{(21)} \text {. }\end{array}$ \\
\hline
\end{tabular}

Fuente: Elaboración propia

$\boldsymbol{n}=16$ 
una puerta abierta. El cuerpo sufriente desborda la vida, las mujeres no pudieron recuperar su cuerpo, sensación de fragilidad y la hipersensibilidad a la impresión ${ }^{(13)}$. Otras implicaciones psicosociales: pérdida de independencia, pérdida de uno mismo y pérdida de relaciones ${ }^{(14)}$.

¿Cuál es el proceso de adaptación según el modelo de Roy en los pacientes sometidos a cirugía de tumor cerebral? ?¿cómo se da el proceso de adaptación, modo, autoconcepto, en personas con tumor cerebral que sobreviven a la cirugía?

Sólo uno de los artículos parece responder a estas preguntas y es que al parecer las conductas no adaptativas que experimentan las personas con tumores cerebrales primarios incluyen:(12)

Modo fisiológico: los efectos secundarios de los medicamentos, la exposición a las actividades de la vida diaria, fatiga, cefalea, defectos visuales y problemas neurológicos.

Modo de autoconcepto: estrés causado por sentirse mal e incierto acerca del futuro.

Modo de función de rol: no poder cumplir con el rol de género y la posibilidad de ser abandonados o engañados.

Modo de interdependencia: el deterioro en las relaciones sociales causadas por las condiciones negativas y que se experimentan en los otros dos modos hace que se queden aislados y solos sin pareja, amigos, trabajo o familia.

\section{CONCLUSIÓN}

Para abordar el cuidado a los pacientes sobrevivientes de tumor cerebral, es preciso conocer su proceso de afrontamiento y adaptación, indagar sobre su calidad de vida, depresión, ansiedad, deterioro cognitivo, nivel de incertidumbre, etc. tanto a corto como a largo plazo. Es posible que este proceso sea diferente según el tipo de tumor que tenga, desde glioma, astrocitoma o meningioma, o el tipo de cirugía. Evaluar qué otros factores anteriores a la cirugía pueden influir en el afrontamiento para reincorporarse posteriormente, como calidad de vida, estrés, ansiedad, apoyo psicológico, función física, función neurológica y cognitiva. Se sugiere la realización de estudios sobre las vivencias propias de las personas que pasan por éstas experiencias. Es necesario indagar sobre las intervenciones que mejoren los procesos de afrontamiento en su vida de las personas sobrevivientes a un tumor cerebral, en los aspectos psicológicos, biológicos, sociales, espirituales y ambientales.

\section{CONFLICTO DE INTERESES}

Los autores declaran no tener ningún conflicto de intereses.

\section{FINANCIAMIENTO}

Sin financiamiento.

\section{REFERENCIAS BIBLIOGRÁFICAS}

1. Organización mundial de la Salud (OMS). ¿Que son los trastornos neurológicos? [Internet]. OMS. 2016 [actualizada 2018; citado 4 de Abril de 2018] Disponible en: http://www. who.int/features/qa/55/es/.

2. Central Brain Tumor Registry of the United States. Factsheet [Internet]. CBTRUS 2016 [actualizada 2017; citado 4 de Abril de 2018]. Disponible en http://www.cbtrus.org/factsheet/ factsheet.html

3.De Roble P, Fiest K, Frolkis A, Pringsheim T, Atta C, St Germaine-Smith C, Día L, Lam D, Jette N. The worldwide incidence and prevalence of primary brain tumors: a systematic review and meta-analysis. Neuro-Oncology [Internet]. 2015

[citado 10 de Junio de 2018]; (17)6: 776-783. Disponible: doi: 10.1093/neuonc/ nou283.

4. Ostrom QT, Gittleman H, de Blank PM, Finlay JL, Gurney JG, McKean-Cowdin R, Stearns DS, Wolff JE, Liu M, Wolinsky Y, Kruchko C. American brain tumor association adolescent and young adult primary brain and central nervous system tumors diagnosed in the United States in 2008-2012. Neurooncology [Internet]. 2015 [citado 10 de Junio de 2018]; 18(1): 1-50. Disponible en: https://www.ncbi.nlm.nih.gov/pmc/ articles/ PMC4690545/.

5. Medina A, Polanco P, Tello M, Esparza S. Propuesta de cuidados de enfermería en la adaptación de autoconcepto de adultos mayores con diabetes tipo 2. Revista Española de Enfermería de Salud Mental [Internet]. 2019 [citado 20 de Junio de 2019]; 6(1): 27-32. Disponible en: doi: http://doi. org/10.35761/reesme.2018.6.04

6. Medina-Fernández IA, Gallegos-Torres R, Candila-Celis J. Adaptación del modo de autoconcepto en usuarios con diabetes tipo 2 de una unidad de primer nivel. Enfermería universitaria [Internet]. 2018 [citado 20 de Mayo de 2019]; 15(4): 332- 41. Disponible en: https://doi.org/10.22201/ eneo.23958421e.2018.4.532

7. Baksi A, Dicle A. Examining the effect of education given by roy adaptation model in patients with primary brain tumors on symptoms and coping with stress. Int J Caring Sci [Internet]. 2017 [citado 10 de Mayo de 2018]; 10 (2): 843. Disponible en: doi: 10.5812/ircmj.64635

8. Aramesh Z, Mazloum R, Aghebati N, Mashhadi Nejad H. Comparing the Efficacy of Nursing Care based on ESI Triage and Roy Adaptation Model in Predicting the Need for ICU or Ward Admission among Head Trauma Patients. Journal of 
Manzandaran University of Medical Sciences [Internet]. 2017 [citado 10 de Marzo de 2018]; 27(153): 84-94. Disponible en: http:// jmums.mazums.ac.ir/article-1-9513-en.pdf

9. Ownsworth T, Haslam C. Impact of rehabilitation on selfconcept following traumatic brain injury: An exploratory systematic review of intervention methodology and efficacy. Neuro psychological rehabilitation [Internet]. 2014 [citado 10 de Abril 2018]; 26(1): 1-35. Disponible en: https://doi. org/10.108 0/09602011.2014.977924

10.Meskal I, Gehring K, Rutten GJ, Sitskoorn MM. Cognitive functioning in meningioma patients: a systematic review. J Neurooncol [Internet]. 2015 [citado 10 de Abril de 2018]; 121 (3): 617-25. Disponible en: doi: 10.1007/s11060-014-1679-8.

11. Zamanipoor Najafabadi AH, Peeters MC, Dirven L, Lobatto DJ, Groen JL, Broekman ML, Peerdeman SM, Peul WC, Taphoorn MJ, van Furth WR. Impaired health-related quality of life in meningioma patients - a systematic review. Neuro-

Oncology [Internet]. 2016 [citado 10 de Junio de 2018]; 19(7): 897-907. Disponible en: doi:10.1093/neuonc/now250.

12. Simsek $A B$, Dicle $A$. Examination of the adaptation states of patients with primary brain tumor according to the roy adaptation model: a qualitative research. Journal of Neurological Sciences [Turkish] [Internet]. 2013 [citado 10 de Mayo de 2018]; 34(1): 88-107. Disponible en http://www.jns. dergisi.org/text.php3?id=637.

13. Wenström I, Eriksson LE, Ebbeskog B. Living in a paradox-women's experiences of body and life-world after meningioma surgery. Journal Of Advanced Nursing [Internet]. 2012 [citado 10 de Mayo de 2018]; 68(3): 559-568. Disponible en: doi: 10.1111/j.1365-2648.2011.05757).

14. Lucas MR. Psychosocial implications for the patient with a high-grade glioma. Journal of neurocience Nursing [Internet]. 2010 [citado 10 de Julio de 2018]; 42 (2): 104-108. Disponible en: doi: 10.1097 / JNN.0b013e3181ce5a34.

15. Waagemans $M L$, van Nieuwenhuizen $D$, Dijkstra $M$, Wumkes M, Dirven CM, Leenstra S, Reijneveld JC, Klein M, Stalpers LJ. Long-term impact of cognitive deficits and epilepsy on quality of life in patients with low-grade meningiomas. Neuro-surgery-online [Internet]. 2011[citado 10 de Junio de 2018]; 69(1): 72-79. Disponible en: doi: 10.1227/ NEU.0b013e318212badb.

16. Habets EJ, Kloet A, Walchenbach R, Vecht CJ, Klein M, Taphoorn MJ. Tumour and surgery effects on cognitive functioning in high-grade glioma patients. Acta neurochir [Internet]. 2014 [citado 10 de Junio de 2018]; 156 (8): 14511459. Disponible en: doi: 10.1007 / s00701-014-2115-8.
17. Wolters Gregório G, Ponds RW, Smeets SM, Jonker F, Pouwels CG, Verhey FR, van Heugten CM. Associations between executive functioning, coping, and psychosocial functioning after acquired brain injury. Journal and Clinic Psychology Society [Internet]. 2015 [citado 10 de Junio de 2018]; 54 (3): 291-306. Disponible en: https://doi.org/10.1111/ bjc.12074.

18. Bao Y, Li L, Guan Y, Wang W, Liu Y, Wang P, Huang X, Tao $S$, Wang Y. Prevalence and associated positive psychological variables of anxiety and depression among patients with central nervous system tumors in China: a cross-sectional study. J Neuroncolgy [Internet]. 2017 [citado 10 de Abril de 2018]; 26 (2): 262-269. Disponible en: doi: 10.1002/pon.4128.

19. Holmes AC, Adams SJ, Hall S, Rosenthal MA, Drummond KJ. Liaison psychiatry in a central nervous system tumor service. Neuro-Oncology [Internet]. 2015 [citado 10 de Mayo de 2018]; 2(2): 88-92. Disponible en: https://doi.org/10.1093/ nop/npv00.

20. Lin L, Chien LC, Acquaye AA, Vera-Bolanos E, Gilbert MR, Armstrong TS. Significant predictors of patients' uncertainty in primary brain tumors. J Neuroncolgy 2015 [citado 10 de Abril de 2018]; 122(3). Disponible en doi: 10.1007/s11060-0151756-7.

21. Krupp W, Klein C, Koschny R, Holland H, Seifert V, Meixensberger J. Assessment of neuropsychological parameters and quality of life to evaluate outcome in patients with surgically treated supratentorial meningiomas. Neurosurgery [Internet]. 2009 [citado 10 de Mayo de 2018]; 64 (1): 40-7. Disponible en: doi: 10.1227 / 01.NEU.0000336330.75381.39. 